\title{
Recombinant Fusion Allergens, Cry j 1 and Cry j 2 from Japanese Cedar Pollen, Conjugated with Polyethylene Glycol Potentiate the Attenuation of Cry j 1-Specific IgE Production in Cry j 1-Sensitized Mice and Japanese Cedar Pollen Allergen-Sensitized Monkeys
}

\author{
Takashi Fujimura $^{a}$ Koji Fujinami ${ }^{a, c}$ Ryosuke Ishikawaa, c Minoru Tateno ${ }^{\text {a, c }}$ \\ Yoshio Tahara ${ }^{c}$ Yasushi Okumuraa, c Hisashi Ohtac Hiroyuki Miyazakia, c \\ Masaru Taniguchi ${ }^{\mathrm{a}, \mathrm{b}}$ \\ a RIKEN-TORII Joint Research Team and ${ }^{b}$ Laboratory for Immune Regulation, RIKEN Center for Integrative Medical \\ Sciences (IMS), RIKEN (The Institute of Physical and Chemical Research), Yokohama, and 'Torii Pharmaceutical Co. \\ Ltd., Tokyo, Japan
}

\section{Key Words}

Allergen-specific immunotherapy · Allergic rhinitis · Fusion allergen - Hybrid allergen · IgE - Japanese cedar pollinosis . Polyethylene glycol-conjugated allergen · Th2 response .

Vaccine

\begin{abstract}
Background: Japanese cedar (Cryptomeria japonica) pollinosis is the most prevalent seasonal rhinitis in Japan. A standardized Japanese cedar pollen extract (CPE) containing 1.5$4.2 \mu \mathrm{g}$ of Cry 1 is currently the highest-concentration extract available for allergen-specific immunotherapy (SIT) against this pollinosis. Therefore, we developed a PEGylated fusion protein as a more effective SIT vaccine against Japanese cedar pollinosis. Methods: The fusion protein of major allergens for Japanese cedar pollen, Cry j 1 and Cry j 2, was expressed in Escherichia coli and conjugated with polyethylene glycol (PEG). The purified PEGylated Cry j 1/2 fusion protein (PEG-
\end{abstract}

fusion) was subcutaneously injected four times into Cry j 1sensitized mice and CPE-sensitized monkeys. The mice were then subcutaneously challenged with Cry j 1 and serum levels of Cry j 1-specific immunoglobulin, and the proliferation and cytokine production of splenocytes were analyzed. The monkeys were intranasally challenged with CPE and analyzed for Cry j 1-specific immunoglobulin levels in plasma. Results: Cryj 1-specific IgE was significantly attenuated in the PEG-fusion-treated group after Cry j 1-challenge and Cry j 1-specific IgG was significantly increased following PEG-fusion treatment in mice and monkeys. Proliferation and Th2type cytokine production in splenocytes stimulated with Cry j 1 were also reduced in PEG-fusion-treated mice. IL10 and IL2 production were reduced, but not significantly, while IFN- $\gamma$ was significantly increased in the PEG-fusion-treated group. Conclusions: A high-dose injection of PEG-fusion appears to be a valid candidate for a safer and more effective vaccine than the conventional SIT extract for Japanese cedar pollinosis.

(c) 2015 S. Karger AG, Basel

\section{KARGER 125}

(c) 2015 S. Karger AG, Base

$1018-2438 / 15 / 1681-0032 \$ 39.50 / 0$

E-Mail karger@karger.com

www.karger.com/iaa
Correspondence to: Dr. Takashi Fujimura

Laboratory for Vaccine Design, RIKEN Center for Integrative Medical Sciences RIKEN (The Institute of Physical and Chemical Research)

1-7-22 Suehiro-cho, Tsurumi-ku, Yokohama 230-0045 (Japan)

E-Mail takashi.fujimura@ riken.jp 


\section{Introduction}

Japanese cedar pollinosis is one of the most common forms of seasonal allergic rhinitis in Japan. Japanese cedar pollen scatters in early spring, usually from February to the end of April, and many Japanese wear facemasks in order to avoid inhalation of Japanese cedar pollen in the open air [1]. The number of patients allergic to Japanese cedar pollen has been increasing in association with the increasing counts of scattering Japanese cedar pollen; the prevalence of patients suffering from Japanese cedar pollinosis was $26.5 \%$ in 2008 , and thereafter has increased at a rate of approximately $10 \%$ per decade [2].

Seven allergens which reacted with IgE from patients with Japanese cedar pollinosis have been isolated and reported as major or minor allergens for Japanese cedar pollinosis, namely pectate lyase as Cry $\mathrm{j} 1$, polygalacturonase as Cry j 2, thaumatin-like protein as Cry j 3, type IV chitinase, isoflavone reductase-like protein, lipid transfer protein homolog, aspartic protease and subtilisin-like serine protease [3]. Among them, Cry j 1 and Cry $\mathrm{j} 2$ are considered to be major allergens for Japanese cedar pollinosis, with more than $90 \%$ of patients having specific IgEs for both allergens in their serum [4-6]. The Japanese cedar pollen extract (CPE) used for allergenspecific immunotherapy (SIT) has been standardized for the amount of Cry j 1 in Japan [7]. A 2,000-JAU (Japanese allergy unit)/ml extract of standardized CPE is the highest-concentration extract available as a vaccine for subcutaneous and sublingual clinical immunotherapy in Japan. However, this extract contains 1.5-4.2 $\mu \mathrm{g}$ of Cry $\mathrm{j} 1$, whereas the recommended dose for effective subcutaneous immunotherapy is $5-20 \mu \mathrm{g}$ of a major allergen per injection [8]. Therefore, a more concentrated standardized CPE available for clinical use in patients with Japanese cedar pollinosis or a purified or recombinant Cry j 1 and Cry j 2 will be necessary for more effective SIT in Japan.

A therapeutic approach using recombinant allergens for SIT has been carried out for pollinosis and allergy to house dust mites over the last few decades [9]. In this approach, a native or mutated form of major allergens or hypoallergens with sequences shuffled relative to the native allergen is generated as an oligomeric or a hybrid protein by using a recombinant technique. The advantages of using such recombinant allergens as a vaccine for SIT are: (1) this approach would allow the production of a uniform allergen, (2) the vaccine would not need to contain minor allergens, nonallergic proteins or inactive ingredients, (3) a large amount of allergen could be pro-

Attenuation of Cry j 1-Specific IgE

Production after Cry j 1 Challenge duced without natural sources, and (4) the destruction of the secondary and tertiary structure would reduce the occurrence of adverse events. In vitro studies using human peripheral blood mononuclear cells (PBMCs) have revealed that fusion (hybrid) proteins react with $\mathrm{T}$ or $\mathrm{B}$ cell receptors and, thus, would be suitable for future use as SIT vaccines. The studies have also shown that these proteins have the same or higher capacity for $\mathrm{T}$ cell activation and the same or lower ability to bind to serum IgE $[10,11]$.

We develop PEGylated fusion protein with the concept that high-dose injections of the PEGylated fusion protein would prevent antigen-specific IgE production and Th2 responses after allergen provocation. Therefore, we planned to analyze the potential of PEGylated fusion protein to downregulate antigen-specific IgE and Th2 responses using a systemic sensitization model in mice using purified major allergen. As we suspected that mechanisms for allergic rhinitis may be different between mice and humans, e.g. dependency on mast cells and neuropeptidelike neuropeptide such as substance $P$, we analyzed clinical effects by PEGylated protein in monkeys with an intranasal allergen provocation model using crude extract.

This is the first report to show the potency of a PEGylated fusion protein for reducing antigen-specific IgE and Th2 responses in vivo. In this report, we propose that a PEGylated fusion protein conjugated with major allergens, Cry j 1 and Cry j 2, would be advantageous for use as a vaccine for injection SIT in patients with Japanese cedar pollinosis. The PEGylated Cry j $1 / 2$ fusion protein [polyethylene glycol (PEG)-fusion] reduced the induction of Cry j 1-specific IgE following Cry $\mathrm{j} 1$ challenge in Cry $\mathrm{j} 1$-sensitized mice and CPE-sensitized monkeys, and downregulated the activation of Cry $\mathrm{j} 1$-specific Th2 responses in mice. We propose that PEG-fusion is a suitable candidate for further evaluation in humans as a safer and more effective vaccine for SIT in patients suffering from Japanese cedar pollinosis.

\section{Materials and Methods}

\section{Animals}

Six-week-old female BALB/c mice were purchased from Charles River Laboratories Japan Inc. (Yokohama, Japan). All mice were housed under specific pathogen-free conditions. All the experiments using mice were carried out using protocols approved by the Animal Studies Committee of RIKEN Yokohama Institute. Monkeys (Macaca fascicularis) were provided by Primate Agora (Aichi, Japan) and the experiments using monkeys were performed at I Tech Lab. Co. Ltd. (Gifu, Japan), using a protocol that followed their own guidelines for animal experiments. 
Fig. 1. a Amino acid composition of the Cry j $1 / 2$ fusion protein. The serine residues, which were substituted for the cysteine residues in the native Cry $j 1$ and Cry j 2, are indicated in bold with underlining. The C-terminal cysteine residue, which was used for conjugation with PEG, is shown in italics with underlining. The lesions for native Cry j 1, Cry j 2 and Histag are indicated. b An image from the SDS-PAGE for purification of the PEGCry j $1 / 2$ fusion protein. Lane MK shows the protein weight marker. The solubilized inclusion body (lane I) was PEGylated on Ni-ion mobilized resin and then eluted with imidazole (lane $\mathrm{Ni}$ ). The eluted protein was loaded onto a gel filtration chromatography column (lane G). After dialyzing in endotoxin-free PBS, the PEGylated Cry $\mathrm{j} 1 / 2$ fusion protein was sterilized using a $0.22-\mu \mathrm{m}$ membrane filter and used for vaccination (lane $\mathrm{P}$ ); $0.5 \mu \mathrm{g}$ of total protein was loaded onto each lane on $5-20 \%$ linear gradient polyacrylamide gel (Atto Corp., Tokyo, Japan).
$1 \rightarrow$ Cry j 1

MDNPIDSSWRGDSNWAQNRMKLADSAVGFGSSTMGGKGGDLYTVTNSDDDPVN

PAPGTLRYGATRDRPLWIIFSGNMNIKLKMPMYIAGYKTFDGRGAQVYIGNGGPS

VFIKRVSNVIIHGLHLYGSSTSVLGNVLINESFGVEPVHPQDGDALTLRTATNIWID

HNSFSNSSDGLVDVTLSSTGVTISNNLFFNHHKVMLLGHDDAYSDDKSMKVTVA

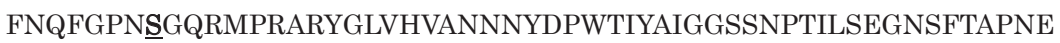

SYKKQVTIRIGS్SKTSSSSSSNWVWQSTQDVFYNGAYFVSSGKYEGGNIYTKKEAFN $I \rightarrow$ Cry j 2

VENGNATPQLTKNAGVLTSSSLSKR $\underline{C}$ RKVEHSRHDAINIFNVEKYGAVGDGKHDS

TEAFSTAWQAASKKKPSAMLLVPGNKKFVVNNLFFNGPSQPHFTFKVDGIIAAYQNP

ASWKNNRIWLQFAKLTGFTLMGKGVIDGQGKQWWAGQSKKWVNGREISNNDRDRP

TAIKFDFSTGLIIQGLKLMNSPEFHLVFGNㅌEGVKIIGISITAPRDSPNTDGIDIFAS

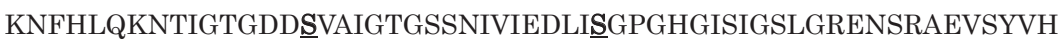

VNGAKFIDTQNGLRIKTWQGGSGMASHIIYENVEMINSENPILINQFYSTTSASASQ

NQRSAVQIQDVTYKNIRGTSATAAAIQLKSSDSMPS్SDIKLSDISLKLTSGKIASSLL $1 \rightarrow$ His tag

a NDNANGYFSGHVIPASKKLSPSHHHHHH

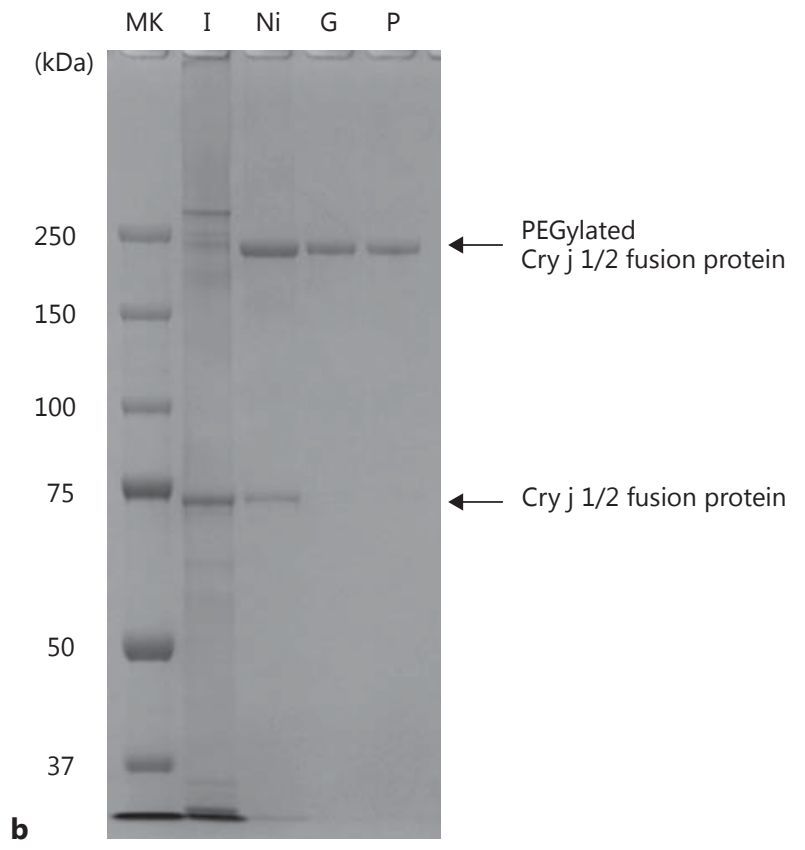

\section{Reagents}

Purified Cry j 1 solution was provided by Torii Pharmaceutical Co. Ltd. (Tokyo, Japan). The Cry j 1 solution contained $2.8 \mathrm{mg} / \mathrm{ml}$ purified Cry $\mathrm{j} 1$ and $0.1 \mathrm{mEU}$ endotoxin per microgram of protein in phosphate-buffered saline (PBS). The Cry $\mathrm{j} 1$ solution was ster- ilized using a 0.22- $\mu \mathrm{m}$ membrane filter (Merck Millipore, Darmstadt, Germany) and stored at $-20^{\circ} \mathrm{C}$ until use. All the chemicals used were of analytical or biochemical grade and purchased from Wako Pure Chemical Industries Ltd. (Osaka, Japan) unless otherwise indicated. 
Synthesis of PEG-Fusion

Complementary DNA for the mutated Cry j 1/2 fusion protein was provided by Dr. Yasuyuki Ishii at RIKEN (Yokohama, Japan). Briefly, cDNA coding for the fusion protein was generated using PCR with Cry j 1 and Cry j 2 (accession numbers AB081309 and AB211810, respectively) as templates. All cysteine residues except a C-terminal cysteine in Cry j 1 and all cysteine residues in Cry j 2 were replaced with serine residues using conventional PCR methods. The mutated Cry $\mathrm{j} 1$ and Cry $\mathrm{j} 2$ were fused in tandem and the resulting fusion protein with a C-terminal His-tag was expressed in Escherichia coli BL21 star (DE3; Invitrogen, Grand Island, N.Y., USA). The obtained inclusion body was solubilized in $4.0 \mathrm{M}$ guanidine- $\mathrm{HCl}$ buffer ( $\mathrm{pH} 8.0$ ) containing $5.0 \mathrm{~mm}$ tris(2-carboxyethyl)phosphine and mixed with Profinity ${ }^{\mathrm{TM}}$ IMAC resin (Bio-Rad Laboratories Inc., Hercules, Calif., USA) for $30 \mathrm{~min}$ at room temperature. The resin was washed with $50 \mathrm{~mm}$ phosphate buffer containing $4.0 \mathrm{M}$ guanidine- $\mathrm{HCl}(\mathrm{pH} 8.0)$ three times and then supplemented with $0.5 \mathrm{mM}$ a-[3-(3-maleimido-1-oxopropyl)amino]propyl- $\omega$-methoxy, polyoxyethylene (Sunbright ME-300MA; NOF Corp., Tokyo, Japan) to attach $30 \mathrm{kDa}$ PEG via a cysteine residue in the C-terminal of the Cry $\mathrm{j} 1$ region (fig. 1a). After mixing overnight at room temperature, the resultant PEG-fusion was eluted from the resin by adding $50 \mathrm{~mm}$ of phosphate buffer containing $4.0 \mathrm{M}$ guanidine- $\mathrm{HCl}$ and $500 \mathrm{~mm}$ imidazole ( $\mathrm{pH} \mathrm{8.0)}$ ), dialyzed against PBS, and then subjected to gel filtration chromatography using a Hiprep 26/60 Sephacryl S-400 HR column (GE Healthcare Japan Ltd., Tokyo, Japan). The resultant fractions containing PEG-fusion were collected, concentrated using a spin column (Amicon Ultra Ultracel-30K; Merck Millipore) and applied to two additional rounds of gel filtration chromatography. The purified PEG-fusion was dialyzed against endotoxin-free PBS and loaded onto a Detoxi-Gel ${ }^{\mathrm{TM}}$ Endotoxin Removing column (Thermo Scientific, Rockford, Ill., USA), and then the concentration of endotoxin was measured by enzyme-linked immunosorbent assay (ELISA; Toxicolor; Seikagaku Corp., Tokyo, Japan) with a Chromogenic Diazo-coupling kit (PyroColor-MP; Seikagaku Corp.) following the manufacturer's protocol. Finally, the PEG-fusion was sterilized with a $0.22-\mu \mathrm{m}$ membrane filter and stored at $-80^{\circ} \mathrm{C}$ until use. The resultant PEG-fusion contained $2.06 \mathrm{mg} / \mathrm{ml}$ protein and $0.045 \mathrm{mEU}$ endotoxin per microgram of protein.

\section{Immunization of Animals}

$\mathrm{BALB} / \mathrm{c}$ mice were intraperitoneally injected with $5 \mu \mathrm{g}$ protein weight of Cry j 1 with $2 \mathrm{mg}$ of Alum (Alhydrogel; Invivogen, San Diego, Calif., USA) in $200 \mu \mathrm{l}$ of endotoxin-free saline (Otsuka Pharmaceutical Factory Inc., Tokushima, Japan) on day 0 , and 2 weeks later they were subcutaneously injected with $5 \mu \mathrm{g}$ protein weight of Cry j 1 (on day 14). Submandibular blood was collected via a facial vein using an animal lancet (MEDIpoint International Inc., Mineola, N.Y., USA) on days 20, 26, 36, 43 and 50. The peripheral blood was incubated at $4^{\circ} \mathrm{C}$ for $1 \mathrm{~h}$, then centrifuged at $12,000 \mathrm{~g}$ for $10 \mathrm{~min}$ at $4^{\circ} \mathrm{C}$, and the serum was obtained. The sera were stored at $-20^{\circ} \mathrm{C}$ until analysis. After quantitation of the Cry j 1-specific IgE concentration by ELISA on days 20 and 26, mice with negative Cry j 1-specific IgE were omitted on day 20 and then, among the remaining group of all sensitized mice on day 26 , mice in which the serum concentration of Cry $\mathrm{j} 1$-specific IgE was between [average - standard deviation (SD)] and (average $+2 \times$ SD) were used for further experiments and randomly divided into groups with almost equal average values and dispersion of Cry j 1-specific IgE by a researcher without using a randomization computer program. Various doses of PEG-fusion or endotoxinfree saline were administered subcutaneously every other day for a total of four times on days $28,30,32$ and 34 . The mice were further sensitized subcutaneously with $5 \mu \mathrm{g}$ of Cry $\mathrm{j} 1$ in $200 \mu \mathrm{l}$ of endotoxin-free saline 3 days after the last vaccination (on day 37 ). The peripheral blood was collected on days 43 and 50, and the serum Cry j 1-specific IgE concentration was determined. The mice were sacrificed 1 week after the last bleeding (on day 57; fig. 2a).

Five monkeys were sensitized by subcutaneous injection of $0.5 \mathrm{ml}$ of CPE with an equal amount of Imject ${ }^{\circledR}$ Alum solution (Thermo Scientific) containing $0.1-0.5 \mu \mathrm{g}$ of Cry j 1 and $20 \mathrm{mg} / \mathrm{ml}$ of aluminum hydroxide and magnesium hydroxide on days -63 and -28 . The sensitization was performed under anesthesia with $100 \mathrm{mg} / \mathrm{kg}$ medetomidine hydrochloride (Kyouritsu Seiyaku Corp., Tokyo, Japan) and $2.5 \mathrm{mg} / \mathrm{kg}$ ketamine hydrochloride (Daiichi Sankyo Propharma Co. Ltd., Tokyo, Japan) and was repeated twice. After confirming that the monkeys were positive for Cry $j$ 1-specific IgE by ELISA and showed positive nasal discharge after intranasal challenge with CPE on day -21, the monkeys were challenged with $0.2 \mathrm{ml}$ per nostril of CPE containing $5 \mu \mathrm{g} / \mathrm{ml}$ Cry j 1 under anesthesia with $10 \mathrm{mg} / \mathrm{kg}$ ketamine hydrochloride weekly; this challenge was also performed on days $-7,12$ and 32. Nasal discharge scores in the right and left anterior naris were scored 20 min after intranasal challenge with CPE on days -7 and 32 . The score was determined as the nasal discharge score according to the Practical Guideline for the Management of Allergic Rhinitis in Japan (PG-MARJ): normal fluid = 0; viscous fluid = 1 ; serous fluid = 2. One milligram of PEG-fusion was subcutaneously injected once per week for 4 weeks (on days 1, 8, 15 and 22) and the animals were challenged with $0.2 \mathrm{ml}$ per nostril of CPE containing $5 \mu \mathrm{g} / \mathrm{ml}$ Cry j 1 on days 12 and 32. Peripheral blood was obtained on days -21 , $-7,12$ and 32 from the saphenous vein under anesthesia with $10 \mathrm{mg} / \mathrm{kg}$ ketamine hydrochloride (fig. 2b).

\section{ELISA for Specific IgE to Cry 1 and IgG Inhibition Assay}

The levels of IgE specific to Cry j 1 were measured by fluorometric ELISA [12]. Briefly, $100 \mu \mathrm{l}$ of $2 \mu \mathrm{g} / \mathrm{ml}$ monoclonal antibody to mouse IgE (clone 6HD5; Yamasa Corp., Tokyo, Japan) or $1 \mu \mathrm{g} /$ $\mathrm{ml}$ goat anti-human IgE ( $\varepsilon$ chain) unconjugated (Life Technologies, Thermo Fisher Scientific Inc., Waltham, Mass., USA) in $0.2 \mathrm{M}$ bicarbonate buffer ( $\mathrm{pH} 9.5$ ) was applied to a 96-well microtiter plate (NUNC-Immuno ${ }^{\circledR}$ Maxisorp; Thermo Fisher Scientific Inc.) and incubated at room temperature for $2 \mathrm{~h}$. After the plate was blocked with PBS containing $0.5 \%$ bovine serum albumin (BSA; Sigma-Aldrich Co. LLC, St. Louis, Mo., USA), 5\% fetal cow serum (FCS; MP Biomedicals, LLC, Solon, Ohio, USA), 0.02\% sodium azide and $0.05 \%$ polyoxyethylene (20) sorbitan monolaurate at room temperature for $1 \mathrm{~h}, 100 \mu \mathrm{l}$ of 160 -fold diluted sera from mice or 200-fold diluted plasma from monkeys in PBS containing $10 \%$ FCS was added to the wells, and the plate was incubated overnight at $4^{\circ} \mathrm{C}$. Then, $100 \mu \mathrm{l}$ of $0.2 \mu \mathrm{g} / \mathrm{ml}$ biotin-labeled Cry j 1 (Hayashibara Biochemical Laboratories Inc., Okayama, Japan) in PBS containing 1\% BSA was added after the plates were washed 3 times with PBS containing $0.1 \%$ polyoxyethylene (20) sorbitan monolaurate (Cry j 1-specific IgE assay) or left unwashed (IgG inhibition assay) [13]. Next, the plates were incubated at room temperature for $1 \mathrm{~h}$, followed by the addition of $100 \mu \mathrm{l}$ of 5,000-fold diluted streptavidin- $\beta$-galactosidase-conjugate (Roche Diagnostics $\mathrm{GmbH}$, Mannheim, Germany) to the wells and additional incuba- 


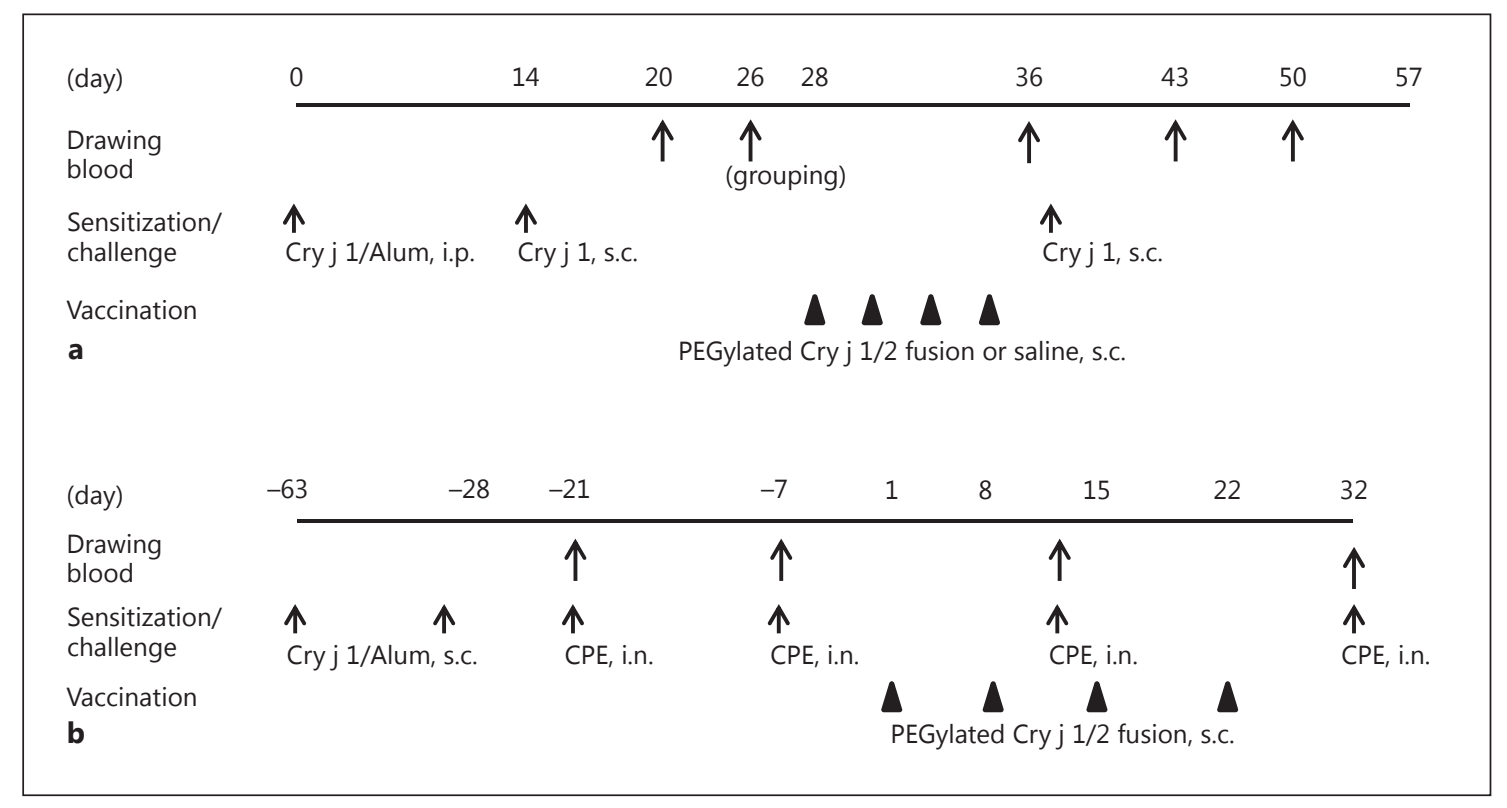

Fig. 2. Experimental procedures for the animal treatment of mice (a) and monkeys (b). Details are given in the Materials and Methods section. Eight mice were used for the immunoglobulin and proliferation assays, 5 mice were used for the cytokine assay and 5 monkeys were used for the immunoglobulin assay. i.p. = Intraperitoneal; s.c. $=$ subcutaneous; i.n. $=$ intranasal.

tion at room temperature for $1 \mathrm{~h}$. For the enzymatic reaction, 0.2 $\mathrm{mM}$ of 4 -methylumbelliferyl $\beta$-D-galactopyranoside (Sigma-Aldrich Japan KK, Tokyo, Japan) was added and the plates were incubated at $37^{\circ} \mathrm{C}$ for $2 \mathrm{~h}$. After stopping the reaction with $0.1 \mathrm{M}$ glycine- $\mathrm{NaOH}$ ( $\mathrm{pH}$ 10.2), the fluorescence intensity was measured using a fluorometric microplate reader (ARVO X3; PerkinElmer Inc., Waltham, Mass., USA). The serum concentration of mouse Cry j 1-specific IgE was calculated on a standard curve generated using a reference anti-Cry j 1 monoclonal IgE which was kindly provided by Dr. Yasuyuki Ishii (RIKEN). The plasma titer of monkey Cry j 1-specific IgE was calculated on a standard curve generated using pooled monkey plasma as $100 \mathrm{kAU}$ (arbitrary units).

\section{ELISA for IgGs Specific to Cry $j 1$}

One hundred microliters of 0.5 or $2 \mu \mathrm{g} / \mathrm{ml}$ Cry j 1 in $0.2 \mathrm{M} \mathrm{bi-}$ carbonate buffer ( $\mathrm{pH}$ 9.5) was applied to a 96 -well microtiter plate for mouse or monkey IgG ELISA, respectively, and then the plate was incubated overnight at $4^{\circ} \mathrm{C}$. After the plate was blocked with PBS containing $0.5 \%$ BSA, $5 \%$ FCS, $0.02 \%$ sodium azide and $0.05 \%$ polyoxyethylene (20) sorbitan monolaurate at room temperature for $1 \mathrm{~h}, 100 \mu \mathrm{l}$ of 50,000- or 100,000-fold diluted sera from mice, or 24,000-fold diluted plasma from monkeys, was added to the wells and the plate was incubated at room temperature for $1 \mathrm{~h}$. Then, $100 \mu$ of 20,000-fold diluted affinity-purified antibody peroxidase-labeled goat anti-mouse IgG (KPL Inc., Gaithersburg, Md., USA) or 10,000-fold diluted anti-monkey IgG (whole molecule) peroxidase antibody produced in rabbits (Sigma-Aldrich) was added, and the plate was incubated at room temperature for $1 \mathrm{~h}$. For the enzymatic reaction, $100 \mu \mathrm{l}$ of TMB ELISA substrate solution (eBioscience Inc., San Diego, Calif., USA) was added and the plate was incubated for $3 \mathrm{~min}$ at room temperature. After the reaction was stopped by adding $25 \mu \mathrm{l}$ of $1.0 \mathrm{M}$ sulfuric acid, the absorbance at $450 \mathrm{~nm}$ was measured using a microplate reader. The serum concentration of mouse Cry j 1-specific IgG was calculated on a standard curve generated for a reference anti-Cry j 1 monoclonal IgG provided by Torii Pharmaceutical Co. Ltd. The plasma titer of monkey Cry j 1-specific IgG was calculated on a standard curve generated using pooled monkey plasma as $100 \mathrm{kAU}$.

\section{Cytokine and Proliferation Assays of Splenocytes from}

\section{Immunized Mice}

$1 \times 10^{6}$ splenocytes from sensitized mice were cultured with or without $5 \mu \mathrm{g} / \mathrm{ml}$ Cry j 1 in $200 \mu \mathrm{l}$ RPMI medium (Sigma-Aldrich) containing 10\% FCS (Gibco, Invitrogen, Grand Island, N.Y., USA), $50 \mu \mathrm{M}$ 2-mercaptoethanol, 100 units/ml penicillin (Sigma-Aldrich), and $100 \mu \mathrm{g} / \mathrm{ml}$ streptomycin (Sigma-Aldrich) for 3 days. After the culture, $1 \mu \mathrm{Ci}(37 \mathrm{kBq})$ per well of [methyl- $\left.{ }^{3} \mathrm{H}\right]$-thymidine (PerkinElmer) was added and the cells were cultured for an additional $18 \mathrm{~h}$. The cells were harvested and incorporation of ${ }^{3} \mathrm{H}$ thymidine was counted for 1 min using a MicroBeta microplate counter (PerkinElmer). For the cytokine assay, plates that had been cultured for 3 days were centrifuged at $300 \mathrm{~g}$ for $10 \mathrm{~min}$ at room temperature, and the supernatant was stored at $-20^{\circ} \mathrm{C}$ until analysis. The concentrations of interleukin (IL)2, IL5, IL10, IL12, IL13 and IFN- $\gamma$ in the culture supernatant were then quantitated using a $\mathrm{BD}^{\mathrm{TM}}$ Cytometric bead assay (BD Biosciences, San Diego, Calif., USA) following the manufacturer's instructions.

\section{Data Representation and Statistical Analysis}

Representative data from three independent experiments using 8 mice for Cry j 1-specific immunoglobulin and proliferation assays and 5 mice for cytokine assay are shown. Nonrepeated ANO-
Fujimura et al. 
VA was applied to compare data among multiple groups and, when the difference was significant, post hoc analysis using a Student-Newman-Keuls (SNK) test was performed. For comparisons between two groups, an unpaired Student t test or Wilcoxon $t$ test was used. Statistical significance was defined as $\mathrm{p}<0.05$.

\section{Results}

\section{Characterization of PEG-Fusion Vaccine}

For future application as a SIT vaccine for allergic patients with Japanese cedar pollinosis, two major allergens from the pollen, namely Cry j 1 and Cry j 2, were expressed as a fusion protein in E. coli. The His-tag at the C-terminal end of the fusion protein was essential for purification using nickel immobilized affinity resin (data not shown). To improve the solubility of the fusion protein, PEG was covalently conjugated via a cysteine residue at the C-terminal of the Cry 1 region (italicized character with underlining in fig. 1a). The PEG-fusion was purified from inclusion bodies of $E$. coli by nickel affinity resin and further purified by gel filtration chromatography. After purification by nickel affinity resin, the ratio of PEG-fusion to the non-PEGylated fusion protein was 77.5:22.5, as estimated by densitometry analysis for the image of SDS-PAGE (lane Ni in fig. 2b). The resulting PEG-fusion was $250 \mathrm{kDa}$ on SDS-PAGE and consisted of $95.8 \%$ of total component proteins. The remaining $4.2 \%$ was the non-PEG-fusion, which was $75 \mathrm{kDa}$ on the SDS-PAGE (lane $\mathrm{P}$ in fig. 2b). Conjugation of the $30-\mathrm{kDa}$ PEG with a $75-\mathrm{kDa}$ fusion protein may prevent electrophoresis by hydrophilic interaction with acrylamide gel or shield the surface electrostatic charge, and the conjugated protein appeared at $250 \mathrm{kDa}$ on SDS-PAGE [14]. Conformation of native Cry j 1 and Cry j 2 was considered to be destroyed in fusion protein, but we did not analyze the structure of the fusion protein by circular dichroism spectroscopy or nuclear magnetic resonance. The resultant PEG-fusion protein had much less binding capacity with anti-Cry j 1 and anti-Cry j 2 monoclonal and polyclonal antibody because of modification with PEG and, in part, destruction of conformational B cell epitopes of Cry $j 1$ and Cry j 2 (data not shown).

\section{Systematic Sensitization Model in Mice}

We first analyzed the capacity of PEG-fusion to downregulate the induction of Cry $\mathrm{j}$ 1-specific IgE following subcutaneous challenge with Cry j 1 . Cry j 1-sensitized mice were subcutaneously injected with 100,20 or $4 \mu \mathrm{g}$ of PEG-fusion per mouse, and each mouse was injected four

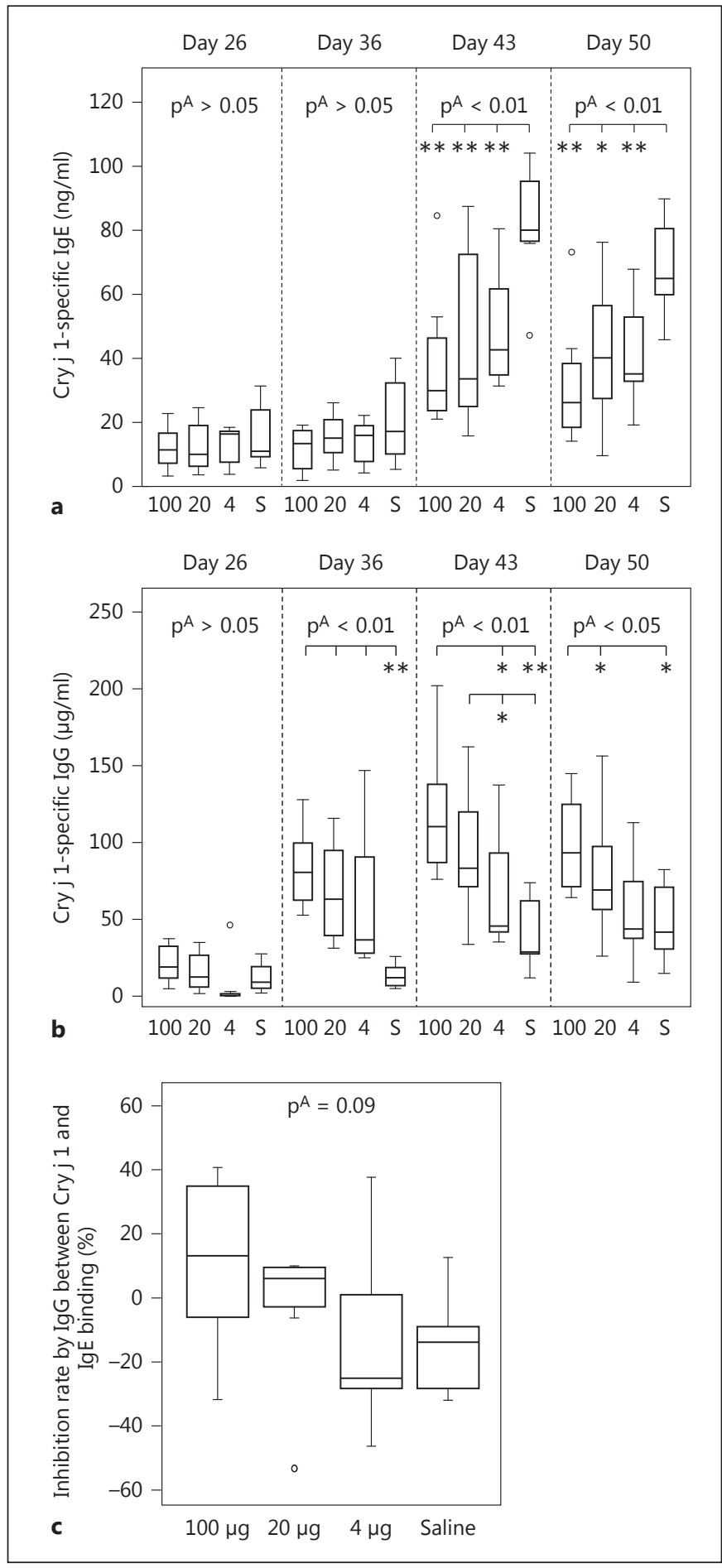

Fig. 3. Changes of the Cry j 1-specific IgE and IgG concentration throughout the study. The individual Cry j 1-specific IgE (a) and Cry j 1 -specific IgG (b) concentrations of 8 mice in each group are indicated using a box plot. $\mathrm{c}$ The inhibition of binding between Cry j 1 and serum IgE by serum IgG on day 43 is shown using a box plot as the inhibition rate. $\mathrm{p}^{\mathrm{A}}=\mathrm{p}$ values estimated by nonrepeated ANOVA. Significant differences as estimated by post hoc SNK test: ${ }^{*} \mathrm{p}<0.05,{ }^{* *} \mathrm{p}<0.01$. 


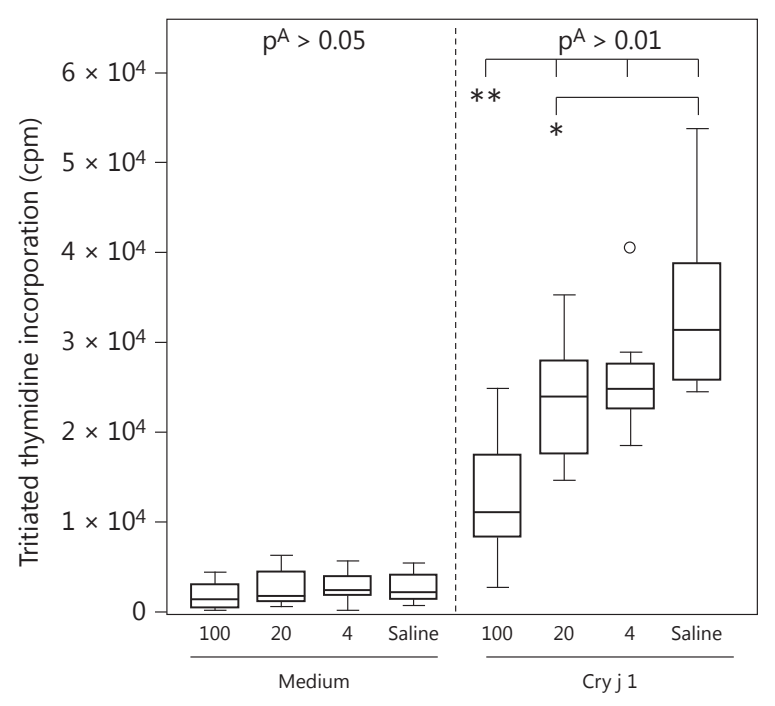

Fig. 4. Splenocytes from the PEGylated Cry j $1 / 2$ fusion proteinand saline-treated mice were cultured with or without Cry $j 1$ for 3 days (shown as Cry j 1 and Medium, respectively). The tritiated thymidine incorporation over the last $18 \mathrm{~h}$ of culture was counted and plotted. $\mathrm{p}^{\mathrm{A}}=\mathrm{p}$ values estimated by nonrepeated ANOVA. Significant differences as estimated by post hoc SNK test: ${ }^{*} \mathrm{p}<0.05$, ** $\mathrm{p}<0.01$.

times with the same dose. The mice were then challenged with $5 \mu \mathrm{g}$ of Cry j 1 subcutaneously 2 days after the last vaccination. The Cry j 1-specific IgE concentration before Cry j 1-challenge was comparable among the groups (day 36) irrespective of whether or not PEG-fusion was administered. However, 1 week after the Cry $j 1$ challenge, the Cry j 1-specific IgE concentration was significantly attenuated in the PEG-fusion-treated groups in a dosedependent manner compared to that in the saline-treated group (fig. 3a). The serum Cry j 1-specific IgG concentration was significantly increased after the PEG-fusion injection compared with that in the saline-treated group (fig. 3b). The Cry j 1-specific IgG concentration was further increased after challenge with Cry $j 1$ in a dose-dependent manner, and that from the 100- $\mu$ g PEG-fusiontreated group was significantly higher than those from the 4- $\mu \mathrm{g}$ - and saline-treated groups on day 43 or those from the 20- $\mu \mathrm{g}$ - and saline-treated groups on day 50 (fig. 3b). The ratio of inhibition by serum IgG for binding between Cry $\mathrm{j} 1$ and serum IgE was highest in the $100-\mu \mathrm{g}$ PEG-fusion-treated group relative to that in the salinetreated group and the degree of inhibition tended to increase in a dose-dependent manner, although there were no statistically significant differences among the groups by nonrepeated ANOVA (fig. 3c). In the two-group comparisons, the ratio of inhibition was significantly different between the 100- $\mu$ g- and saline-treated groups by the unpaired Student $t$ test $(\mathrm{p}<0.05)$. The induction of inhibitory IgG may prevent IgE-facilitated antigen presentation by dendritic cells and B cells to antigen-specific Th2 cells and also prevent activation of basophils and mast cells after systematic challenge with Cry $\mathrm{j} 1$ [15].

The Cry j 1-stimulated proliferation of splenocytes was significantly reduced in the 100- and 20- $\mu$ g PEG-fusiontreated groups compared with those in the saline-treated group. Among the PEG-fusion-treated groups, proliferation in the 100- $\mu$ g PEG-fusion-treated group was significantly reduced compared with the other groups (fig. 4). Therefore, we decided to use $100 \mu \mathrm{g}$ of PEG-fusion as the most effective dose for the cytokine production assay, as judged from its potential to reduce IgE production and cell proliferation.

The cytokine productions from Cry $\mathrm{j}$ 1-stimulated splenocytes of mice in the 100- $\mu$ g PEG-fusion- and saline-treated groups were analyzed. IFN- $\gamma$ production was significantly increased in the PEG-fusion-treated group, and the levels of production of Th2-type cytokines, such as IL5 and IL13, were significantly decreased in the PEGfusion-treated group compared to those in the salinetreated group. IL10 production also tended to be decreased in the PEG-fusion-treated group, and the level of IL2 production was slightly decreased but roughly comparable between the PEG-fusion- and saline-treated groups (fig. 5). These data suggested that the Cry j 1-specific Th2 responses were attenuated by the induction of a Cry j 1-specific Th1 response or Cry j 1-specific T cell anergy. The precise mechanism underlying the attenuation of the Th2 response remains unclear, and thus further analysis will be needed to elucidate the mechanisms for attenuation of both the Cry j 1-specific IgE and Th2 responses by vaccination with PEG-fusion.

\section{Nasal Provocation Model in Monkeys}

We next confirmed the potential of PEG-fusion as a vaccine for SIT to attenuate Cry j 1-specific IgE in cynomolgus monkeys sensitized with CPE (fig. 2b). In this preliminary study, 5 monkeys sensitized with CPE were injected with PEG-fusion and the changes of immunoglobulin titers from those before vaccination and intranasal Cry $\mathrm{j} 1$ challenge were measured. It would have been preferable to use saline-treated monkeys as a control, but additional monkeys were not available. A decrease of antigen-specific IgE or attenuation of symptoms after SIT
38

Int Arch Allergy Immunol 2015;168:32-43 DOI: $10.1159 / 000441141$
Fujimura et al. 


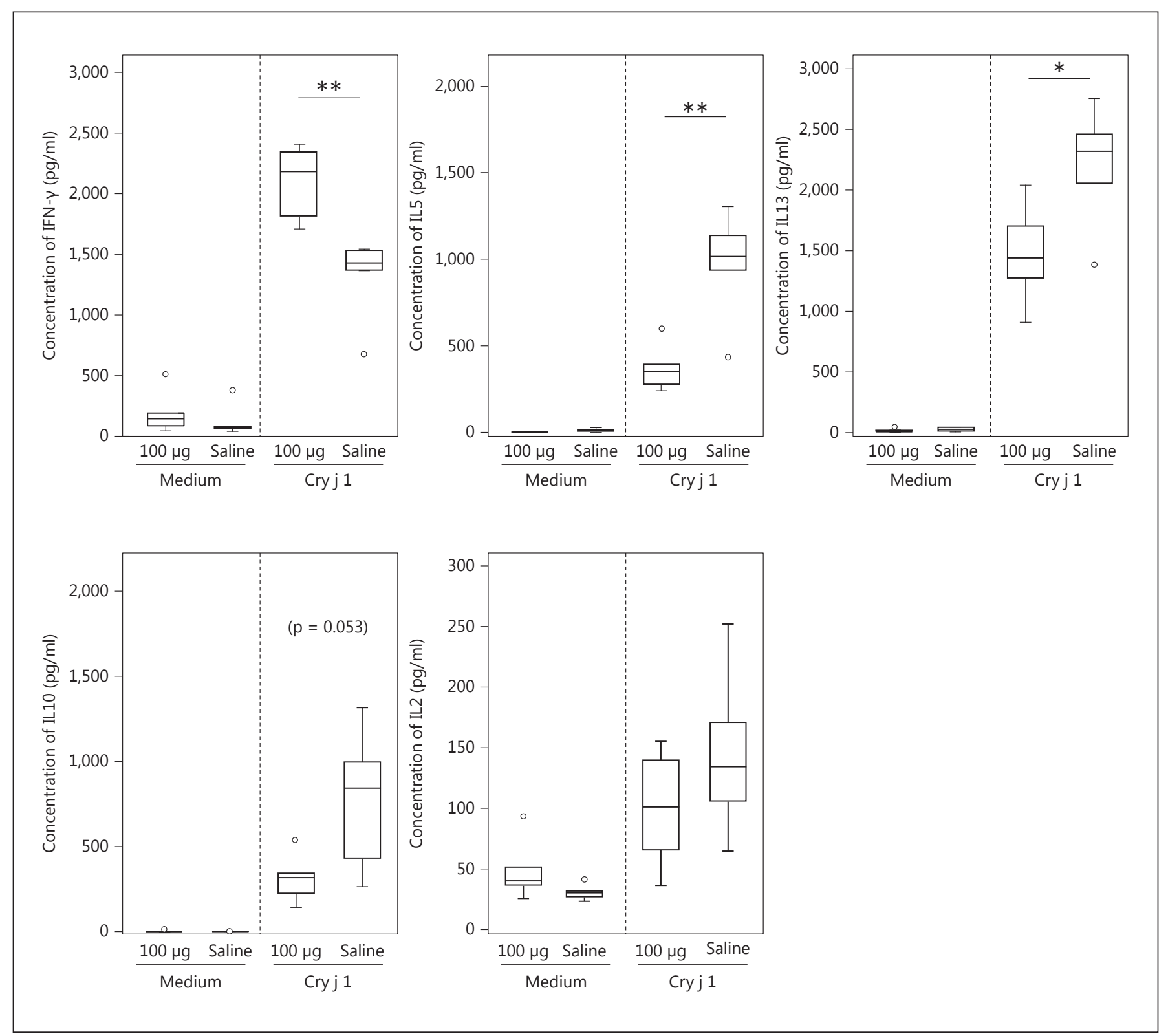

Fig. 5. Cytokine concentration of the culture supernatant from splenocytes stimulated with Cry j 1 for 3 days. Whole splenocytes from mice treated with the $100 \mu \mathrm{g}$ of PEGylated Cry $\mathrm{j} 1 / 2$ fusion protein or saline were cultured with or without Cry $j 1$ (shown as
Cry j 1 and Medium, respectively). Each group includes the data from 5 mice. Significant differences as estimated by the unpaired Student $t$ test: ${ }^{*} \mathrm{p}<0.05,{ }^{* *} \mathrm{p}<0.01$. and the following pollen season compared with those before vaccination has been reported in preliminary clinical studies in humans $[16,17]$. In the present experiments in monkeys, after four injections of PEG-fusion as a vaccine, the levels of Cry j 1-specific IgE were significantly decreased in all animals compared to those before vaccination, in spite of the intranasal challenges with CPE during and after the vaccination (fig. 6a, c). On the other hand, Cry j 1-specific IgG was significantly increased after four vaccinations and two intranasal challenges with $\mathrm{CPE}$ (fig. 6b, d). The two injections of PEG-fusion did not induce statistically significant changes in either the Cry j 1-specific IgE or IgG titer compared to those before the injections, but four injections induced a significant 


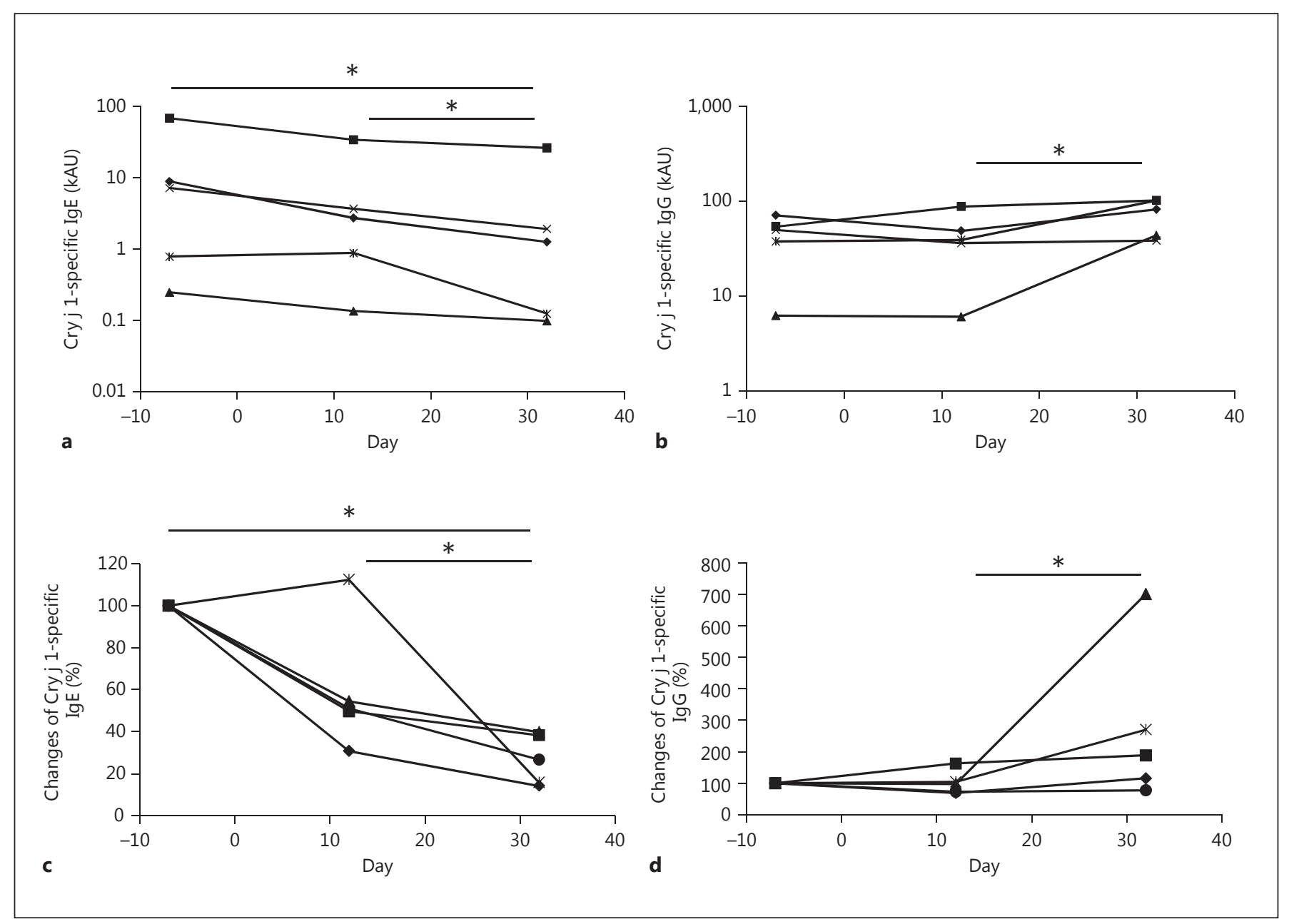

Fig. 6. Titers and percentage changes in the Cry j 1-specific IgE (a, c) and IgG (b, d) titers are shown. The Cry j 1-specific IgE and IgG levels in plasma before vaccination (day -7) were determined and taken as $100 \%$ (c, d). The vaccines were subcutaneously injected four times on days 1, 8, 15 and 22. The monkeys were intranasally challenged with CPE on days 12 and 32 during and after the vaccinations. Each symbol shows the Cry j 1-specific immunoglobulin titer in an individual monkey. Significant differences as estimated by the Wilcoxon $t$ test: ${ }^{*} \mathrm{p}<0.05$. change in the Cry j 1-specific immunoglobulin titer. These data suggest that more than three injections of PEG-fusion are needed to modify the immunoglobulin profiles in monkeys, and probably also in humans. The decrease of Cry $\mathrm{j}$ 1-specific IgE and increase of Cry j 1-specific IgG after four vaccinations in the CPE-sensitized monkeys was similar to that observed in the Cry j 1-sensitized mice.

We also analyzed nasal discharge scores after provocation with $\mathrm{CPE}$ as a clinical score to show the therapeutic effects of PEG-fusion. The scores for nasal discharge were significantly attenuated after four injections of PEG-fusion concomitant with a decrease of Cry $\mathrm{j} 1$-specific $\operatorname{IgE}$ and increase of Cry j 1-specific IgG (table 1). Further analyses to clarify the therapeutic effects of PEG-fusion in placebo-controlled studies in monkeys and humans are needed.

\section{Discussion}

Japanese cedar pollen contains large amounts of polysaccharides and glycoproteins. This composition makes it difficult to produce a high concentration of stable extract such as would be suitable for clinical use. For a more effective SIT using a higher dose of the major allergens
40

Int Arch Allergy Immunol 2015;168:32-43 DOI: $10.1159 / 000441141$
Fujimura et al. 
Table 1. Scores for nasal discharge in the right and left nostrils of monkeys before and after vaccination

\begin{tabular}{|c|c|c|c|c|c|c|c|c|c|c|c|}
\hline \multirow[b]{3}{*}{ Before } & \multicolumn{10}{|c|}{ Nasal discharge scores in each individual monkey } & \multirow[t]{2}{*}{ Median } \\
\hline & \multicolumn{5}{|c|}{ left nostril } & \multicolumn{5}{|c|}{ right nostril } & \\
\hline & 1 & 2 & 2 & 2 & 2 & 1 & 2 & 2 & 2 & 2 & 2 \\
\hline After & 1 & 1 & 1 & 2 & 1 & 0 & 0 & 1 & 2 & 1 & $1^{*}$ \\
\hline
\end{tabular}

Scoring was carried out before and after vaccination on days -7 (before) and 32 (after). Significant difference as estimated by the Wilcoxon $t$ test: ${ }^{*} \mathrm{p}<0.05$.

from Japanese cedar pollen, the best approaches would seem to be either purifying the native major allergens or generating a recombinant form of the major allergens. However, recombinant Cry j 1 and Cry j 2 produced by microbes are highly insoluble, probably due to the missing or altered polysaccharide chain (data not shown). Therefore, we decided to conjugate PEG with the Cry j 1/2 fusion protein to improve the solubility of the protein. All cysteine residues in the fusion protein except for a cysteine residue in the C-terminal region of Cry $\mathrm{j} 1$ were replaced with serine to regulate the number and loci for PEGylation to achieve quality control of the PEGylated protein as a SIT vaccine. T cell epitopes determined for SIT vaccine do not contain any cysteine residue in Cry j 1 and Cry j 2, or contain only one substituted cysteine residue in the Cry j 2 portion in 7 epitopes from Cry $j 1$ and Cry 2 [18, 19]. Therefore, the substitution of cysteine residue with serine residue does not seem to alter the $\mathrm{T}$ cell reactivity of fusion protein from naive allergens in patients with Japanese cedar pollinosis. PEGylation also increases the safety of the vaccine by preventing binding with specific IgE [20]. Indeed, no fatal or local adverse events, such as anaphylactic shock, swelling or flare at the injection site, were observed in the mice and monkeys in our experiments, even though we used subcutaneous injections of as much as $0.5 \mathrm{mg}$ of PEG-fusion per site into two loci on the back of the monkeys (data not shown). Furthermore, PEGylated allergens have been shown to be more effective at inducing suppressor $\mathrm{T}$ cell responses than the native allergens used in conventional SIT vaccines [21]. Therefore, the PEG-fusion would be a valid candidate for further evaluation in humans as a powerful vaccine for curative treatment against Japanese cedar pollinosis. In our experiments using mice and monkeys, four subcutaneous injections of the PEG-fusion reduced the following Cry j 1-specific IgE production by challenge with Cry $\mathrm{j} 1$ and CPE. The results suggest that PEG-fusion can attenuate the Cry j 1-specific IgE titer and clinical

Attenuation of Cry j 1-Specific IgE

Production after Cry j 1 Challenge symptoms while requiring fewer injections and a shorter vaccination period due to the use of a higher dose of PEGfusion compared to the classical SIT using an unmodified crude standardized extract.

One advantage of using the whole sequence of an allergen molecule is that the complete repertoire of $\mathrm{T}$ cell epitopes can be included. The regions of allergens recognized by $\mathrm{T}$ cells vary among individuals according to the diversity of HLA class II and T cell receptors. A genetically engineered fusion protein of Api $\mathrm{m} 1$ and Api m 2, two major allergens for bee (Apis mellifera) venom, induced basically the same levels of $\mathrm{T}$ cell proliferation and cytokine production in human PBMCs as induced by the native Api $\mathrm{m} 1$ and Api $\mathrm{m} 2$ [11]. In a report using a hybrid molecule of four grass (Phleum pratense) allergens, $\mathrm{Phl} \mathrm{p} \mathrm{1,} \mathrm{Phl} \mathrm{p} \mathrm{2,} \mathrm{Phl} \mathrm{p} 5$ and Phl p 6, the proliferative response of PBMCs from patients allergic to grass allergens stimulated with the recombinant hybrid molecule was significantly higher than those induced by a mixture of the four grass allergens [22]. These reports suggest that hybrid fusion allergens have more potential to activate antigen-specific $\mathrm{T}$ cells compared to conjugated $\mathrm{T}$ cell epitopes, and even compared to a mixture of native allergens.

We used two major allergens from Japanese cedar pollen, Cry j 1 and Cry j 2, in developing our recombinant fusion vaccine for SIT against Japanese cedar pollinosis because most allergic patients with Japanese cedar pollinosis possess reactive $T$ cells and IgEs specific to either Cry j 1 or Cry $\mathrm{j} 2$ in their peripheral blood [23]. It has been reported that subcutaneous injection of conventional Japanese cedar pollen allergen reduced specific IgE and Th2 responses after more than 3 years of treatment [24]. PEG-fusion reduced specific IgE after four injections in mice and monkeys, and specific Th2 responses in mice. Although additional experiments will be needed to optimize the dose and frequency of injection and obtain the most effective clinical outcome by this vaccination, our 
data from mice and monkeys suggest that shorter and less frequent injections may also be effective to reduce CPEspecific IgE and Th2 responses in humans compared to the protocol when using a conventional standardized extract. The optimal dose and the number of injections should be examined in phase II clinical trials in humans.

In this study, four injections with PEG-fusion attenuated the proliferation and Th2-type cytokine productions in splenocytes stimulated with Cry j 1 (fig. 4, 5). IFN- $\gamma$ production was significantly higher in the PEG-fusiontreated group than in the saline-treated group. IL12 production was undetectable in either the PEG-fusion- or saline-treated group after stimulation with Cry 1 in vitro (data not shown). These data suggest that PEG-fusion SCIT induced Cry j 1-specific Th1 responses. Such suppression of Th2-type responses and increase of Th1-type responses induced by SIT were similar in a human study using PBMCs $[24,25]$. On the other hand, many recent reports have shown the induction of suppressor or regulatory T cells after SIT in human clinical trials $[26,27]$. However, in our study, both IL2 and IL10 production were comparable between the PEG-fusion- and salinetreated groups, although proliferation was significantly suppressed in the PEG-fusion-treated group (fig. 4). This suggested induction of $\mathrm{T}$ cell anergy rather than induction of IL10-producing cells by PEG-fusion treatment. However, we were unable to exclude the possibility that IL10-producing regulatory $T$ cells or B cells would be induced by PEG-fusion treatment, although IL10 was undetectable in the culture supernatant [28]. The precise mechanism by which PEG-fusion treatment reduced the
Th2-type responses and proliferation of splenocytes should be elucidated in a future human clinical study.

We suggested a positive therapeutic effect after four rounds of injection of PEG-fusion determined by the nasal discharge score (table 1). In this preliminary study, we compared the score between pre- and posttreatment of the vaccine as changes from baseline. We should confirm the therapeutic effects in a placebo-controlled study using a greater number of monkeys. Furthermore, we need to analyze the optimal dose and safety of PEG-fusion, involving higher doses in monkeys, to set up a phase II clinical trial in humans.

In conclusion, the PEG-fusion is a suitable candidate for a safer and more effective SIT vaccine as a curative treatment against Japanese cedar pollinosis.

\section{Acknowledgements}

We sincerely thank Dr. Toshitada Takemori, Dr. Takashi Saito, Dr. Masato Kubo, Dr. Hisahiro Yoshida, Dr. Hiroshi Ohno and Dr. Yasuyuki Ishii (RIKEN, Yokohama, Japan) for their helpful comments and fruitful discussions. We also sincerely thank Ms. Hisako Motokawa and Akiko Imai (RIKEN, Yokohama, Japan) for their excellent technical and administrative assistance.

\section{Disclosure Statement}

This work was a collaborative effort between RIKEN and Torii pharmaceutical Co. Ltd., and was financially supported by Torii Pharmaceutical Co. Ltd. None of the authors have any other potential conflicts of interest to report.

\section{References}

1 Yamada T, Saito H, Fujieda S: Present state of Japanese cedar pollinosis: the national affliction. J Allergy Clin Immunol 2013;133:632-639.e5.

2 Saito Y: Japanese cedar pollinosis: discovery, nomenclature, and epidemiological trends. Proc Jpn Acad Ser B Phys Biol Sci 2014;90:203210.

3 Fujimura T, Kawamoto S: Spectrum of allergens for Japanese cedar pollinosis and impact of component-resolved diagnosis on allergen-specific immunotherapy. Allergol Int 2015;64:312-320.

4 Fujimura T, Shigeta S, Kawamoto S, Aki T, Masubuchi M, Hayashi T, Yoshizato K, Ono K: Two-dimensional IgE-binding spectrum of Japanese cedar (Cryptomeria japonica) pollen allergens. Int Arch Allergy Immunol 2004;133:125135.

5 Yasueda H, Yui Y, Shimizu T, Shida T: Isolation and partial characterization of the major allergen from Japanese cedar (Cryptomeria japonica) pollen. J Allergy Clin Immunol 1983;71:77-86

6 Sakaguchi M, Inouye S, Taniai M, Ando S, Usui M, Matuhasi T: Identification of the second major allergen of Japanese cedar pollen. Allergy 1990;45:309-312.

7 Yasue $\mathrm{H}$, Okuda M, Yoshida $\mathrm{H}$, Ito K, Baba M, Iikura Y, Okuhira H, Inoue S, Sakaguchi $\mathrm{M}$, Miyamoto A: Guidelines for standardization of allergens and cedar pollen allergen extract in Japan (in Japanese). Arerugi 1996;45: 416-421.

8 Cox L, Nelson H, Lockey R, Calabria C, Chacko T, Finegold I, Nelson M, Weber R, Bernstein DI, Blessing-Moore J, Khan DA, Lang DM, Nicklas RA, Oppenheimer J, Portnoy JM, Randolph C, Schuller DE, Spector SL, Tilles S, Wallace D: Allergen immunotherapy: a practice parameter third update. J Allergy Clin Immunol 2011;127:S1-S55.

9 Bhalla PL, Singh MB: Biotechnology-based allergy diagnosis and vaccination. Trends Biotechnol 2008;26:153-161.

10 Bonura A, Corinti S, Artale A, Di Felice G Amoroso S, Melis M, Geraci D, Colombo P: A hybrid expressing genetically engineered major allergens of the Parietaria pollen as a tool for specific allergy vaccination. Int Arch Allergy Immunol 2007;142:274-284.

11 Kussebi F, Karamloo F, Rhyner C, SchmidGrendelmeier P, Salagianni M, Mannhart C, Akdis M, Soldatova L, Markovic-Housley Z, von Beust BR, Kundig T, Kemeny DM, Blaser $\mathrm{K}$, Crameri R, Akdis CA: A major allergen gene-fusion protein for potential usage in allergen-specific immunotherapy. J Allergy Clin Immunol 2005;115:323-329. 
12 Toda M, Sato H, Takebe Y, Taniguchi Y, Saito S, Inouye S, Takemori T, Sakaguchi M: Inhibition of immunoglobulin E response to Japanese cedar pollen allergen (Cry j 1) in mice by DNA immunization: different outcomes dependent on the plasmid DNA inoculation method. Immunology 2000;99:179-186.

13 Wurtzen PA, Lund G, Lund K, Arvidsson M, Rak S, Ipsen H: A double-blind placebo-controlled birch allergy vaccination study II: correlation between inhibition of IgE binding, histamine release and facilitated allergen presentation. Clin Exp Allergy 2008;38:12901301.

14 Fee CJ, van Alstine JM: Purification of pegylated proteins. Methods Biochem Anal 2011;54:339-362.

15 Kepil Ozdemir S, Sin BA, Guloglu D, Ikinciogullari A, Gencturk Z, Misirligil Z: Shortterm preseasonal immunotherapy: is early clinical efficacy related to the basophil response? Int Arch Allergy Immunol 2014; 164 237-245.

16 Cingi C, Aynaci S, Cakli H, Cingi E, Ozudogru E, Kecik C, Altin F, Bal C: Efficacy of longterm sublingual-oral immunotherapy in allergic rhinitis. Acta Otorhinolaryngol Ital 2005;25:214-219.

17 Suarez-Fueyo A, Ramos T, Galan A, Jimeno L, Wurtzen PA, Marin A, de Frutos C, Blanco C, Carrera AC, Barber D, Varona R: Grass tablet sublingual immunotherapy downregulates the $\mathrm{T}_{\mathrm{H}} 2$ cytokine response followed by regulatory $\mathrm{T}$-cell generation. J Allergy Clin Immunol 2014;133:130-138.e1-2.

18 Sone T, Morikubo K, Miyahara M, Komiyama N, Shimizu K, Tsunoo H, Kino K: T cell epitopes in Japanese cedar (Cryptomeria ja- ponica) pollen allergens: choice of major $\mathrm{T}$ cell epitopes in Cry j 1 and Cry j 2 toward design of the peptide-based immunotherapeutics for the management of Japanese cedar pollinosis. J Immunol 1998;161:448-457.

19 Hirahara K, Tatsuta T, Takatori T, Ohtsuki M, Kirinaka H, Kawaguchi J, Serizawa N, Taniguchi Y, Saito S, Sakaguchi M, Inouye S, Shiraishi A: Preclinical evaluation of an immunotherapeutic peptide comprising $7 \mathrm{~T}$-cell determinants of Cry $j 1$ and Cry $j 2$, the major Japanese cedar pollen allergens. J Allergy Clin Immunol 2001;108:94-100.

20 Lee WY, Sehon AH: Suppression of reaginic antibodies with modified allergens. I. Reduction in allergenicity of protein allergens by conjugation to polyethylene glycol. Int Arch Allergy Appl Immunol 1978;56:159-170.

21 Lee WY, Sehon AH, Akerblom E: Suppression of reaginic antibodies with modified allergens. IV. Induction of suppressor T cells by conjugates of polyethylene glycol (PEG) and monomethoxy PEG with ovalbumin. Int Arch Allergy Appl Immunol 1981;64:100114.

22 Linhart B, Hartl A, Jahn-Schmid B, Verdino P, Keller W, Krauth MT, Valent P, Horak F, Wiedermann U, Thalhamer J, Ebner C, Kraft D, Valenta R: A hybrid molecule resembling the epitope spectrum of grass pollen for allergy vaccination. J Allergy Clin Immunol 2005;115:1010-1016.

23 Sugimura K, Hashiguchi S, Takahashi Y, Hino K, Taniguchi Y, Kurimoto M, Fukuda K, Ohyama M, Yamada G: Th1/Th2 response profiles to the major allergens Cry $\mathrm{j} 1$ and Cry j 2 of Japanese cedar pollen. Allergy 1996;51: $732-740$.
24 Ohashi Y, Nakai Y, Kakinoki Y, Ohno Y, Tanaka A, Masamoto T, Sakamoto H, Washio Y, Kato A: Immunotherapy affects the seasonal increase in specific IgE and interleukin-4 in serum of patients with seasonal allergic rhinitis. Scand J Immunol 1997;46:6777.

25 Schulten V, Tripple V, Sidney J, Greenbaum J, Frazier A, Alam R, Broide D, Peters B, Sette A: Association between specific timothy grass antigens and changes in $\mathrm{T}_{\mathrm{H}} 1$ - and $\mathrm{T}_{\mathrm{H}}$ 2-cell responses following specific immunotherapy. J Allergy Clin Immunol 2014;134:1076-1083.

26 Fujimura T, Yonekura S, Horiguchi S, Taniguchi $\mathrm{Y}$, Saito $\mathrm{A}$, Yasueda $\mathrm{H}$, Inamine $\mathrm{A}, \mathrm{Na}-$ kayama T, Takemori T, Taniguchi M, Sakaguchi M, Okamoto Y: Increase of regulatory T cells and the ratio of specific IgE to total $\operatorname{IgE}$ are candidates for response monitoring or prognostic biomarkers in 2-year sublingual immunotherapy (SLIT) for Japanese cedar pollinosis. Clin Immunol 2011;139:65-74.

27 Bohle B, Kinaciyan T, Gerstmayr M, Radakovics A, Jahn-Schmid B, Ebner C: Sublingual immunotherapy induces IL-10-producing $\mathrm{T}$ regulatory cells, allergen-specific $\mathrm{T}$-cell tolerance, and immune deviation. J Allergy Clin Immunol 2007;120:707-713.

28 Fujimura T, Yonekura S, Taniguchi Y, Horiguchi S, Saito A, Yasueda H, Nakayama T, Takemori T, Taniguchi M, Sakaguchi $M$, Okamoto Y: The induced regulatory $\mathrm{T}$ cell level, defined as the proportion of IL$10^{+} \mathrm{Foxp}^{+}$cells among $\mathrm{CD} 25^{+} \mathrm{CD} 4^{+}$leukocytes, is a potential therapeutic biomarker for sublingual immunotherapy: a preliminary report. Int Arch Allergy Immunol 2010;153: 378-387. 